\title{
A robust prognostic gene expression signature for early stage lung adenocarcinoma
}

\author{
Marcin Krzystanek ${ }^{*}$, Judit Moldvay ${ }^{2}$, David Szüts ${ }^{3}$, Zoltan Szallasi ${ }^{1,4,5}$ and Aron Charles Eklund ${ }^{1}$
}

\begin{abstract}
Background: Stage I lung adenocarcinoma is usually not treated with adjuvant chemotherapy; however, around half of these patients do not survive 5 years. Therefore, a reliable prognostic biomarker for early stage patients would be critical to identify those most likely to benefit from early additional treatments. Several studies have searched for gene expression prognostic biomarkers for lung adenocarcinoma, but these have not yielded a widely accepted prognosticator.

Results: We analyzed gene expression from seven published lung adenocarcinoma cohorts for which we included only stage I and II patients who were not given adjuvant therapy. Seven genes consistently obtained statistical significance in Cox regression for overall survival. The combined signature has a weighted mean hazard ratio of 3.2 in all cohorts and 3.0 (C.I. 1.3-7.4, p<0.01) in an independent validation cohort and is strongly correlated with previously published signatures of chromosomal instability and cell cycle progression.

Conclusions: The new prognostic signature, if validated prospectively, may enable better stratification and treatment of early stage lung cancer patients.
\end{abstract}

Keywords: Lung adenocarcinoma, Prognostic biomarker, Gene expression, Early stage cancer

\section{Background}

Lung cancer has the third highest incidence rate and the highest mortality rate of all cancer types. For non-small cell lung cancer (NSCLC), the 5-year survival rate remains below $15 \%[1,2]$. Given the difficulties with treatment of advanced NSCLC, the most promising possibility of improving outcomes may be efficient diagnosis and treatment of early stage cases. One of the most important clinical decisions in these patients is whether to give adjuvant chemotherapy in addition to surgical resection. At present postoperative chemotherapy is not recommended for patients with completely resected stage IA NSCLC with $1 \mathrm{~A}$ level of evidence, and can be considered in stage IB disease and a primary tumor $>4 \mathrm{~cm}$ with $2 \mathrm{~B}$ level of evidence [2]. Nonetheless, only up to $73 \%$ of stage IA and $58 \%$ of stage IB patients survive 5 years [1]. Therefore, identification of patients who are likely to

\footnotetext{
* Correspondence: marcin@cbs.dtu.dk

${ }^{1}$ Department of Systems Biology, Center for Biological Sequence Analysis,

Technical University of Denmark, Lyngby, Denmark

Full list of author information is available at the end of the article
}

benefit from adjuvant treatment - even with NSCLC of stage I - would be of strong diagnostic and prognostic relevance.

A similar problem has been extensively studied and to a significant extent answered in node negative estrogen receptor positive breast cancer. A gene expression signature was obtained that identifies patients with high risk of recurrence who benefit from additional chemotherapy [3]. Identification of such a gene expression signature often initiates with the quantification of a large number of genes on a patient cohort where outcome is known and then the most informative genes are selected and further validated.

Similar strategies have been applied to lung adenocarcinoma as well. Several cohorts containing early to mid stage lung cancer patients have been subjected to transcriptomic analysis, mainly by microarray [4-10]. However, no reliable, consistent gene expression based prognosticator has emerged from these efforts. One possible reason for this failure could be that some of these studies followed a suboptimal strategy in one or more possible ways: 1) 
searching for a general NSCLC prognosticator, as opposed to lung adenocarcinoma (LUAD)- or lung squamous carcinoma (LUSC)-specific signatures $[4,5], 2)$ doing the analysis in a treated/untreated mixed population $[4,6,9]$, 3 ) ignoring the possibility of technical bias in the microarray data $[11,12]$. On the other hand, a PCR-based gene expression signature of cell cycle progression (CCP), which was not derived from lung cancer patients, was found to be prognostic in three early stage LUAD cohorts and validated in a PCR-based study $[13,14]$.

The increasing availability of lung cancer datasets makes it possible to look for a robust signature comprising of genes that would not otherwise be found if the study had been conducted on only a single dataset. Therefore, we set out to perform a meta-analysis of several lung cancer microarray datasets to see if we could identify a gene expression signature that is prognostic in early stage lung adenocarcinoma patients who were not given chemotherapy.

\section{Methods}

Microarray data were downloaded from the GEO database (GSE8894, GSE14814, GSE30219, GSE31210, GSE37745, GSE50081), except Shedden et al., which was downloaded from caarraydb.nci.nih.gov (Table 1). All calculations were performed using the $\mathrm{R}$ programming language. Each gene expression dataset was normalized with the RMA algorithm except GSE9984, for which we were unable to obtain the original raw data, and therefore we used the version downloaded directly from GEO which had been normalized with the GCRMA algorithm [4]. Each dataset, except GSE9984, was also corrected for two sources of potential bias: the RNA degradation captured as the average decrease in expression between 5 ' probes and 3' probes (degradation bias metric) and the diversity of starting mRNA (RMA IQR bias metric) that remained after RMA normalization. This step was performed using "bias" package version 0.0.5 [11]. Weighted average of hazard ratios was calculated as a mean weighted by size of each dataset.

Table 1 Number of patients in the cohorts included in this study

\begin{tabular}{llllll}
\hline & & \multicolumn{2}{l}{ Included $^{\text {a }}$} & \\
Cohort & Available & Stage I & Stage II & Total \\
\hline Lee et al. 2008 [4] & 138 & 39 & 12 & 51 \\
Zhu et al. 2010 [5] & 133 & 20 & 12 & 32 \\
Shedden et al. 2008 [6] & 505 & 164 & 43 & 207 \\
Rousseaux et al. 2013 [7] & 307 & 81 & 3 & 84 \\
Okayama et al. 2012 [8] & 246 & 162 & 42 & 204 \\
Botling et al. 2013 [9] & 196 & 31 & 4 & 35 \\
Der et al. 2014 [10] & 181 & 92 & 35 & 127 \\
\hline
\end{tabular}

apassed inclusion criteria: adenocarcinoma, no chemotherapy
Gene expression and clinical covariates from the validation cohort were downloaded from the TCGA Data Portal using Data Matrix (https://tcga-data.nci.nih.gov/ tcga/dataAccessMatrix.htm), selecting all available tumors on $15^{\text {th }}$ Jan 2016, disease: LUAD and data type: RNASeqV2. Expression values were extracted from files containing RSEM gene-normalized results and we normalized them across samples using the average gene expression of each tumor $[15,16]$.

\section{Results}

We performed a literature and database search and identified seven publicly available gene expression data sets with overall or recurrence-free survival data and with at least 30 patients meeting the following criteria: 1) profiled on the Affymetrix HG-U133A or HG-U133 Plus 2.0 platform; 2) adenocarcinoma subtype by histological report; 3) pathological stage I or II; and 4) not given neoadjuvant, adjuvant, or targeted therapy (Table 1). Each data set (except Lee et al. [4]) was normalized individually and adjusted to reduce technical bias [11].

To identify genes whose expression level is prognostic, we applied the following procedure to each of the 22,277 common probe sets. We split each cohort into two groups according to the expression value of the probe set, and applied Cox proportional hazards regression and a log-rank test of statistical significance on these two groups. If the hazard ratio had the same directionality in all cohorts, and the P value was below 0.05 in any six of the seven cohorts, the probe set was considered prognostic. We expect this procedure to yield an individual type I error rate of $1.0 \times 10^{-7}$, and with Bonferroni correction for 22,277 probe sets, a family-wise error rate of 0.00036 . This procedure resulted in seven probe sets, each representing a unique gene (Table 2).

The expression values of the seven probe sets were strongly positively correlated, so we defined a prognostic score of an individual tumor as the mean $\log _{2}$ expression value of the seven probe sets (termed "ESLA-7", for early stage lung adenocarcinoma). We found that the ESLA-7 score, when used to stratify each cohort into two groups of equal size, is prognostic in six of the seven individual cohorts (Table 2 and Fig. 1). Notably, the ESLA-7 score was more prognostic than any of the individual probe sets. Overall, the weighted average hazard ratio of ESLA-7 was 3.2, or 3.6 if the Botling cohort was omitted (Fig. 1).

We next performed a multivariate analysis, adjusting for stage, age and stratifying for gender. No other clinical data types were available in all cohorts. In this analysis, ESLA-7 showed a HR ranging between 1.1-4.9 in the individual cohorts, with a weighted mean of 2.7 and statistical significance in all cohorts except Botling et al. 2008 (Table 3). 
Table 2 Hazard ratios for the individual ESLA-7 genes in each cohort

\begin{tabular}{|c|c|c|c|c|c|c|c|c|c|c|c|c|c|c|}
\hline \multirow{3}{*}{$\begin{array}{l}\text { Gene } \\
\text { Probe set } \\
\text { Cohort }\end{array}$} & \multirow{2}{*}{\multicolumn{2}{|c|}{$\frac{\text { ADAM10 }}{202604 \text { __at }}$}} & \multirow{2}{*}{\multicolumn{2}{|c|}{$\frac{\text { DLGAP5 }}{\text { 203764_at }}$}} & \multirow{2}{*}{\multicolumn{2}{|c|}{$\frac{\text { RAD51AP1 }}{\text { 204146_at }}$}} & \multirow{2}{*}{\multicolumn{2}{|c|}{$\frac{\text { FGFR10P }}{205588 \text { s_at }}$}} & \multirow{2}{*}{\multicolumn{2}{|c|}{$\frac{\text { NCGAP }}{218663 \text { at }}$}} & \multirow{2}{*}{\multicolumn{2}{|c|}{$\frac{\text { KIF15 }}{219306 \_a t}$}} & \multirow{2}{*}{\multicolumn{2}{|c|}{$\frac{\text { ASPM }}{219918 \text { _s_at }}$}} \\
\hline & & & & & & & & & & & & & & \\
\hline & $\mathrm{HR}$ & $p$ & $\mathrm{HR}$ & $\mathrm{p}$ & $\mathrm{HR}$ & $\mathrm{p}$ & $\mathrm{HR}$ & $\mathrm{p}$ & $\mathrm{HR}$ & $\mathrm{p}$ & $\mathrm{HR}$ & $p$ & $\mathrm{HR}$ & $\mathrm{p}$ \\
\hline Lee et al. 2008 [4] & 3.0 & ** & 2.3 & * & 4 & *** & 2.9 & ** & 2.3 & * & 2.5 & * & 2.5 & $*$ \\
\hline Zhu et al. 2010 [5] & 0.7 & & 3.1 & * & 3.7 & * & 3.2 & * & 4.7 & $* *$ & 3.9 & * & 4.1 & $* *$ \\
\hline Shedden et al. 2008 [6] & 1.7 & * & 1.7 & * & 1.7 & * & 1.6 & * & 1.8 & * & 1.6 & * & 1.7 & * \\
\hline Rousseaux et al. 2013 [7] & 2.6 & ** & 2.4 & $* *$ & 2.7 & ** & 1.9 & * & 2.3 & ** & 2.3 & $* *$ & 1.9 & $*$ \\
\hline Okayama et al. 2012 [8] & 4.3 & $* * *$ & 2.9 & $* *$ & 2.9 & $* *$ & 3.4 & $* *$ & 2.4 & * & 2.8 & * & 4.2 & *** \\
\hline Botling et al. 2013 [9] & 2.5 & * & 1.7 & & 1.0 & & 1.3 & & 1.3 & & 1.7 & & 1.1 & \\
\hline Der et al. 2014 [10] & 2.2 & $* *$ & 2.5 & $* *$ & 2 & * & 2.1 & $* *$ & 2.5 & $* *$ & 2.4 & $* *$ & 2 & * \\
\hline
\end{tabular}

${ }^{*} p \leq 0.05,{ }^{* *} p \leq 0.01,{ }^{* * *} p \leq 0.001$

To assess the performance of ESLA-7 in an independent cohort that was not used to derive the signature, we analyzed RNA-seq gene expression data from the TCGA LUAD cohort. To reduce the effects of varying chemotherapy regimes in the TCGA cohort, we analyzed recurrence-free survival (RFS) in stage I and II patients from this cohort. The majority of early stage LUAD patients do not receive chemotherapy until recurrence; therefore this analysis could be considered as a more accurate assessment of true prognostic performance. Here, ESLA-7 was statistically significant $(\mathrm{HR}=1.8$, C.I. 1.3-2.6, $\mathrm{p}<0.001$, Additional file 1: Figure S1A). Subsequently, we used available treatment information to censor the survival times at the time of initiation of chemotherapy, thus creating a subcohort of patients who did not receive chemotherapy during the followup period. RFS analysis of stage I and II, untreated patients $(\mathrm{N}=95)$ showed $\mathrm{HR}=$ 3.0 (C.I. 1.3-7.4, $\mathrm{p}<0.01$, Figure 1 and Additional file 1: Figure S1B).

Since most of the seven genes were annotated with functions related to chromosomal instability (CIN), we asked whether we could achieve similar prognostic performance with our previously described CIN25 chromosomal instability signature or with the previously published cell cycle progression (CCP) signature $[13,14,17]$. We applied the CIN25 and the CCP signatures in the same way as the ESLA-7 signature and found that ESLA-7 on average performed better than both CIN25 and CCP (weighted mean hazard ratio of 3.2, 2.9 and 2.8 respectively, Fig. 2). CIN25 and CCP scores also showed trends similar to ESLA-7 when using TCGA LUAD RNAseq data (Additional file 1:

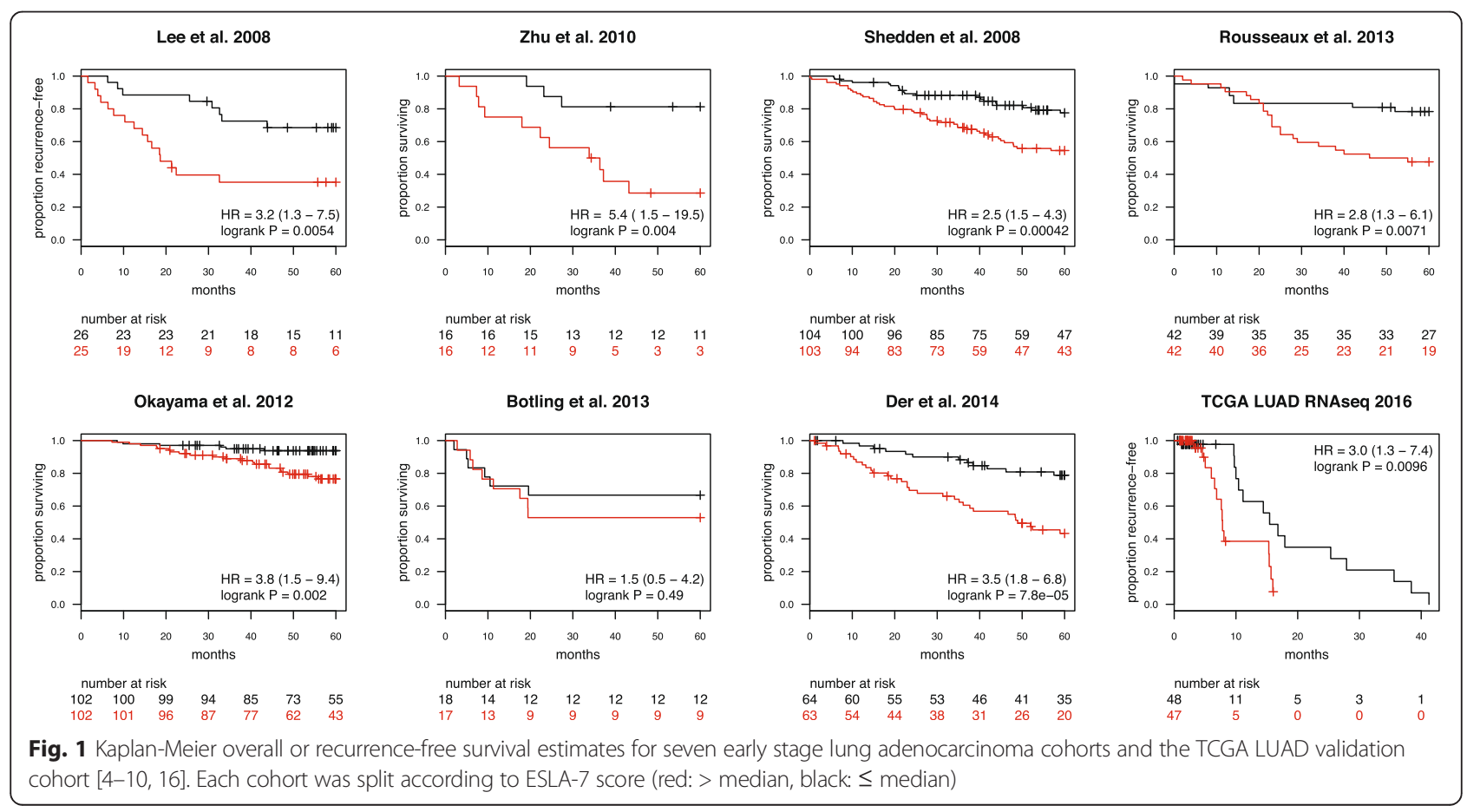


Table 3 Performance of ESLA-7 in multivariate setting when adjusted for age, stage and stratified for gender

\begin{tabular}{llllll}
\hline Cohort & HR & Cl & p & N & events \\
\hline Lee et al. 2008 [4] & 2.9 & $1.2-7.1$ & $*$ & 51 & 24 \\
Zhu et al. 2010 [5] & 4.9 & $1.3-18.1$ & $*$ & 32 & 14 \\
Shedden et al. 2008 [6] & 2.0 & $1.2-3.5$ & $*$ & 207 & 64 \\
Rousseaux et al. 2013 [7] & 2.6 & $1.2-5.8$ & $*$ & 84 & 31 \\
Okayama et al. 2012 [8] & 3.1 & $1.2-7.9$ & $*$ & 204 & 27 \\
Botling et al. 2013 [9] & 1.1 & $0.3-3.9$ & & 35 & 14 \\
Der et al. 2014 [10] & 3.2 & $1.6-6.2$ & $* * *$ & 127 & 44 \\
\hline
\end{tabular}

${ }^{*} p \leq 0.05,{ }^{* *} p \leq 0.01,{ }^{* * *} p \leq 0.001$

Figure S1C-F). Additionally, the correlation between ESLA-7 and CIN25 within each cohort was very high, ranging from 0.88 to 0.98 , suggesting that ESLA-7 to a large extent quantifies CIN. Similarly correlation between ESLA-7 and CCP ranged from 0.89 to 0.99 .

To assess the performance of ESLA-7 without arbitrary stratifying the cohorts into two equally sized groups, we performed Cox regression using ESLA-7 as a continuous variable. Association with overall survival (or recurrencefree survival in the Lee et al. 2008 cohort) was statistically significant in all cohorts except Botling et al. 2013 (weighted mean 2.1, Table 4). Additionally, we explored whether the ESLA-7 score might provide further prognostic stratification into more than two groups, using the ESLA-7 score to stratify each cohort into four groups of equal size. For cohorts with sufficient number of patients in each stratum a clear trend was apparent (Fig. 3). Patients in the first quartile had better survival than patients in the other three quartiles, and, especially, patients in the fourth quartile. No such trend was apparent in the Zhu et al. 2010 [5] and Botling et al. 2013 [9] cohorts, possibly due to the smaller numbers of patients in these two cohorts.

Finally, we applied ESLA-7 to five lung squamous cell carcinoma (LUSC) cohorts [4, 5, 7, 9]. Median split according to ESLA-7 value did not yield statistical significance in any of the LUSC cohorts (Additional file 2: Table S1 and Additional file 3 ). We attempted to create a separate signature for lung squamous cell carcinoma (LUSC). However, similar methodology applied to LUSC patients did not yield a robust expression signature (data not shown).

\section{Discussion}

We used simple methodology to derive a robust prognostic gene expression signature for early stage lung adenocarcinoma. The signature was validated in an independent cohort, however, with 95 patients in the regression, of which many left the study or were censored due to the methodology within the first semester of the follow-up, one should treat this result with caution. If successfully validated in an independent clinical trial, the ESLA-7 signature could potentially be used for guiding clinical onocologist decisions on whether an individual early stage lung adenocarcinoma patient, especially a patient with stage I disease, should receive chemotherapy after surgical resection of the tumor. The seven genes could be combined with an additional small panel of reference genes, as has been done in similar prognostic signatures for other cancer types $[3,14,18]$.

High correlation among ESLA-7 and the two other signatures suggests that to a large degree they may be quantifying the same biological processes. CIN25 was developed as a signature of chromosomal instability from specific genes whose expression was consistently correlated with aneuploidy in several types of tumors. As with CIN25, net overexpression of ESLA-7 was predictive of poor clinical outcome. Both signatures contain genes with function connected directly with kinetochore assembly: KIF20A, KIF4A, TPX2, PRC1, and TTK in CIN25; and KIF15, DLGAP5, and ASPM in ESLA-7. Also, both contain genes that are part of condensin complexes: NCAPG in ESLA-7; and NCAPD2 in CIN25.

\begin{tabular}{|c|c|c|c|c|c|c|c|c|}
\hline \multirow[b]{2}{*}{ Study } & \multirow[b]{2}{*}{ Deaths } & \multicolumn{2}{|l|}{ ESLA-7 } & \multicolumn{2}{|l|}{ CIN25 } & \multicolumn{2}{|l|}{$\mathrm{CCP}$} & \multirow{2}{*}{$\begin{array}{l}\text { ESLA-7 } \\
\text { CIN25 } \\
\text { CCP }\end{array}$} \\
\hline & & HR & $\mathbf{P}$ & HR & $\mathbf{P}$ & HR & $\mathbf{P}$ & \\
\hline Lee et al. 2008 & $26 / 51$ & $3.2(1.3-7.5)$ & 0.0054 & $3.1(1.3-7.3)$ & 0.0065 & $2.4(1.1-5.5)$ & 0.032 & $\because \bar{C}$ \\
\hline Zhu et al. 2010 & $15 / 32$ & $5.4(1.5-19.5)$ & 0.004 & $2.1(0.7-6.3)$ & 0.18 & $2.25(0.75-6.73)$ & 0.14 & \multirow{2}{*}{$\because$} \\
\hline Shedden et al. 2008 & $82 / 207$ & $2.5(1.5-4.3)$ & 0.00042 & $2.2(1.3-3.6)$ & 0.0025 & $2.4(1.4-4)$ & $9 e-04$ & \\
\hline Rousseaux et al. 2013 & $45 / 84$ & $2.8(1.3-6.1)$ & 0.0071 & $2.8(1.3-6.1)$ & 0.0071 & $2.8(1.3-6.1)$ & 0.0071 & $\bar{\Xi}$ \\
\hline Okayama et al. 2012 & $30 / 204$ & $3.8(1.5-9.4)$ & 0.002 & $3.8(1.5-9.3)$ & 0.0021 & $3(1.3-7.2)$ & 0.0081 & $\Longrightarrow$ \\
\hline Botling et al. 2013 & $22 / 35$ & $1.5(0.5-4.2)$ & 0.49 & $0.99(0.35-2.82)$ & 0.98 & $1.5(0.5-4.2)$ & 0.49 & $\Longrightarrow$ \\
\hline Der et al. 2014 & $51 / 127$ & $3.5(1.8-6.8)$ & $7.8 \mathrm{e}-05$ & $3.5(1.8-6.8)$ & $9 e-05$ & $3.6(1.8-6.9)$ & $6.7 \mathrm{e}-05$ & $\equiv$ \\
\hline \multicolumn{2}{|l|}{ Summary } & \multicolumn{2}{|l|}{3.2} & \multicolumn{2}{|l|}{2.9} & \multicolumn{2}{|l|}{2.8} & \multirow[b]{2}{*}{ 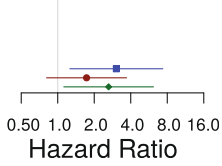 } \\
\hline TCGA LUAD 2016 & $24 / 95$ & $3.0(1.3-7.4)$ & 0.0096 & $1.7(0.8-3.7)$ & 0.15 & $2.6(1.1-6.2)$ & 0.021 & \\
\hline \multicolumn{9}{|c|}{$\begin{array}{l}\text { Fig. } 2 \text { Forest plot indicating the performance of the ESLA-7, CIN25 [17] and CPP [13] signatures in the seven early stage lung adenocarcinoma } \\
\text { cohorts and in the TCGA LUAD validation cohort }\end{array}$} \\
\hline
\end{tabular}


Table 4 Hazard ratios of cox regression using ESLA-7 as continuous variable

\begin{tabular}{llllll}
\hline Cohort & $\mathrm{HR}$ & $\mathrm{Cl}$ & $\mathrm{p}$ & $\mathrm{N}$ & events \\
\hline Lee et al. 2008 [4] & 1.8 & $1.3-2.6$ & $* * *$ & 51 & 24 \\
Zhu et al. 2010 [5] & 3.8 & $1.1-12.1$ & $*$ & 32 & 14 \\
Shedden et al. 2008 [6] & 2.4 & $1.6-3.5$ & $* * *$ & 207 & 64 \\
Rousseaux et al. 2013 [7] & 2.2 & $1.5-3.3$ & $* * *$ & 84 & 31 \\
Okayama et al. 2012 [8] & 1.9 & $1.3-2.8$ & $* * *$ & 204 & 27 \\
Botling et al. 2013 [9] & 1.2 & $0.7-2.2$ & & 35 & 14 \\
Der et al. 2014 [10] & 1.6 & $1.1-2.2$ & $*$ & 127 & 44 \\
\hline
\end{tabular}

${ }^{*} p \leq 0.05,{ }^{* *} p \leq 0.01,{ }^{* * *} p \leq 0.001$

Both kinetochores and condensin complexes play central roles in chromosome assembly and segregation. Overall, both signatures point towards chromosomal instability as an important factor in early stage patient outcome.

Only a single gene, RAD51-associated protein 1 (RAD51AP1), is present in both signatures. This gene plays an important role in homologous recombinationmediated chromosome damage repair by enhancing the recombinase activity of RAD51.

The ESLA-7 signature includes genes ADAM10 and FGFR10P, which have been reported to contribute to oncogenesis of various cancer types including lung cancer, and whose function indicates that they may take part in oncogenic transformation and/or increase cell proliferation by interaction with major signaling cascades (ERK1/2, p38 MAPK, and STAT, Notch1, RAS/MAPK, PLC- $\gamma$, and PI3K/AKT). The slightly better performance of ESLA-7 as compared to CCP might be due to additional information carried by ADAM10 and/or FGFR10P, which are slightly less correlated with the remainder of the ESLA7 genes (Additional file 4: Figure S2).

Interestingly, forkhead box MI (FOXM1), which takes part in the regulation of spindle assembly genes, nearly passed our criteria. This gene is also one of the top CIN25 and CCP genes and, more recently, was identified as predictor of adverse outcomes in various malignancies in a cross-platform, cross-cancer study [19].

While we found a monotonic correlation between the CIN signature and prognosis in LUAD, we previously found a non-monotonic relationship between CIN and prognosis in LUSC, in which very low and very high levels of CIN result in increased survival compared to the rest of the cohort [20].

\section{Conclusions}

If CIN is indeed highly prognostic in early stage lung cancer, as our results indicate, then this would suggest that LUAD and LUSC cohorts should be analyzed separately. The new prognostic signature, if further validated prospectively, will enable better stratification and reduce overtreatment of early stage lung cancer patients.

\section{Statement on ethics approval}

In this study we used publicly available data collected with patients consent approved by relevant institutional review board following declaration of Helsinki.

Data was approved by following institutional boards: Institutional review board of Samsung Medical Center, and written informed consent was obtained (IRB 2005-

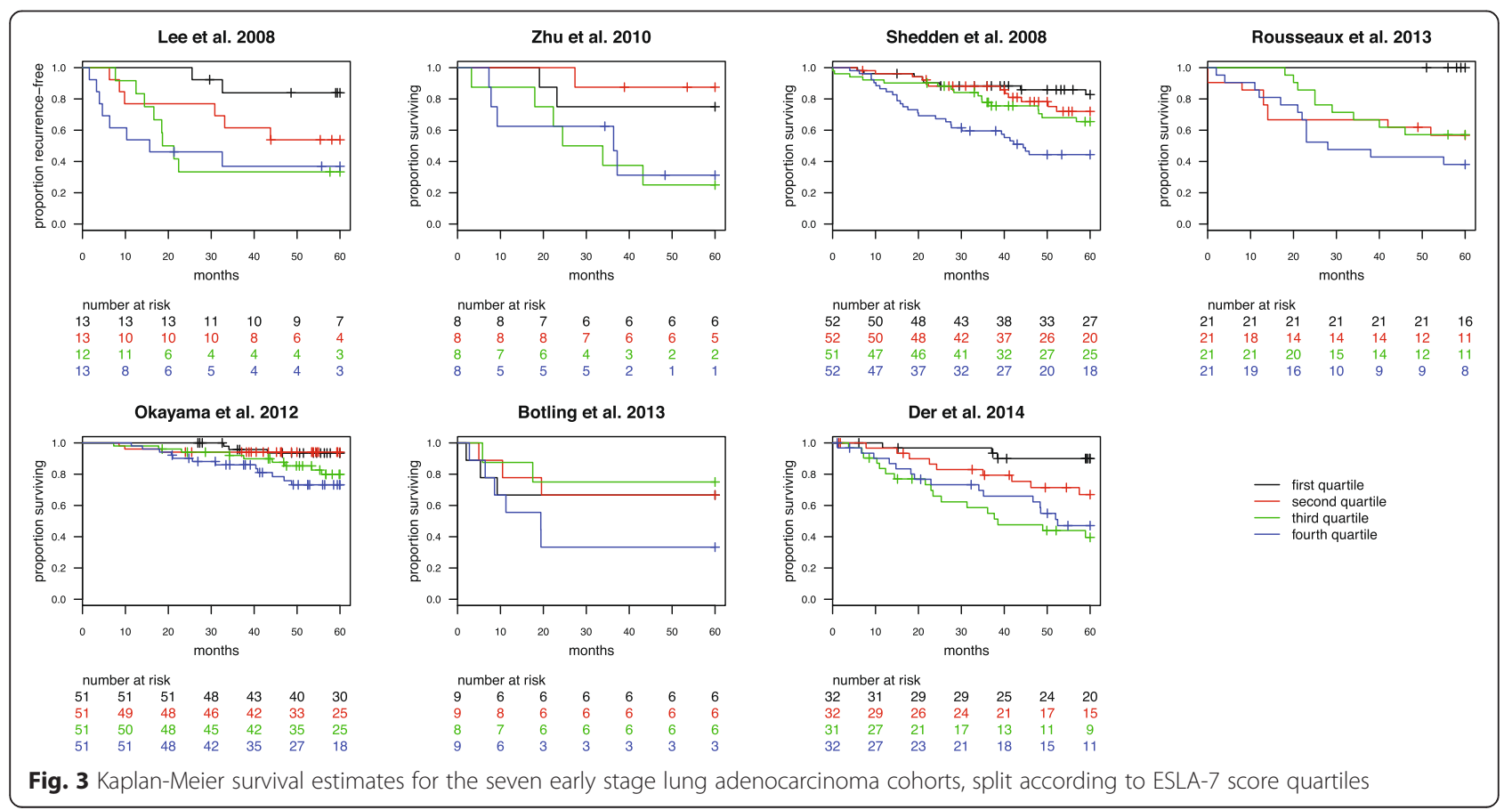


12-034), The University Health Network Research Ethics Board, Institutional Review Board of each of the four institutions' (University of Michigan Cancer Center (UM), Moffitt Cancer Center (HLM), Memorial Sloan-Kettering Cancer Center (MSK) and the Dana-Farber Cancer Institute (CAN/DF)), Institutional Review Board of the National Cancer Center, Tokyo, Japan, Uppsala regional ethical review board, reference \#2006/ 325 and Linkoeping regional ethical review board, reference \#2010/44-31, Research Ethics Board of University Health Network (UHN181), for TCGA data used in this study declaration on ethics is as follows: All specimens were obtained from patients with appropriate consent from the relevant institutional review board.

\section{Additional files}

Additional file 1: Figure S1A. TCGA LUAD RNAseq - stage I and II ESLA - 7, DFS. Figure S1B: TCGA LUAD RNAseq - stage I and II ESLA - 7, DFS - no treatment. Figure S1C: TCGA LUAD RNAseq - stage I and II CIN25, DFS. Figure S1D: TCGA LUAD RNAseq - stage I and II CIN25, DFS - no treatment. Figure S1E: TCGA LUAD RNAseq - stage I and II CCP, DFS. Figure S1F: TCGA LUAD RNAseq - stage I and II CCP, DFS - no treatment. (PDF $20 \mathrm{~kb}$ )

Additional file 2: Table S1. Performance of ESLA-7 in chosen lung squamous datasets. Median split according to ESLA-7 score. ${ }^{*} p \leq 0.05$, ${ }^{* *} \mathrm{p} \leq 0.01,{ }^{* * *} \mathrm{p} \leq 0.001$. (DOCX $59 \mathrm{~kb}$ )

Additional file 3: TCGA LUSC clinical data. (TXT $124 \mathrm{~kb}$ ) Additional file 4: Figure S2. GSE8894 NSCLC Adeno untreated. (PDF $11 \mathrm{~kb}$ )

\section{Abbreviations \\ CCP: cell cycle progression (signature); CIN: chromosomal instability (signature); ESLA: early stage lung adenocarcinoma (signature); GEO: gene expression omnibus; LUAD: lung adenocarcinoma; LUSC: lung squamous carcinoma; NSCLC: non small lung cancer; OS: overall survival; PCR: polymerase chain reaction; RFS: recurrence-free survival.}

\section{Competing interests}

ACE and ZS are named inventors on a patent related to the CIN25 chromosomal instability signature mentioned in this paper. For the remaining authors none were declared.

\section{Authors' contributions}

MK and ZS designed the study. MK, ACE and ZS performed the analysis. MK, ZS, JM, DS and ACE wrote the manuscript. DS, JM contributed to critical review of the manuscript. All authors read and approved the final manuscript.

\section{Funding}

This work was supported by the Danish Council for Independent Research [09-073053/FSS]; the Breast Cancer Research Foundation, the Széchenyi Progam, Hungary [KTIA_NAP_13-2014-0021], MTA Homing Program 2013, MTA-TKI643/ 2012 and the Novo Nordisk Foundation [to ZS]; the Danish Cancer Society grants R72- R90-A6213 [to MK] and A4618-13-S2 [to ACE] and the MTA Momentum Program 2011 [to DS].

\section{Author details}

'Department of Systems Biology, Center for Biological Sequence Analysis, Technical University of Denmark, Lyngby, Denmark. ${ }^{2}$ Department of Tumor Biology, National Koranyi Institute - Semmelweis University, Budapest, Hungary. ${ }^{3}$ Institute of Enzymology, Research Centre for Natural Sciences, Hungarian Academy of Sciences, Budapest, Hungary. ${ }^{4}$ Computational Health Informatics Program (CHIP), Boston Children's Hospital, Boston, USA. ${ }^{5}$ Harvard Medical School, Boston, USA.
Received: 8 December 2015 Accepted: 10 February 2016

Published online: 19 February 2016

\section{References}

1. Crinò L, Weder W, van Meerbeeck J, Felip E. Early stage and locally advanced (non-metastatic) non-small-cell lung cancer: ESMO Clinical Practice Guidelines for diagnosis, treatment and follow-up. Ann Oncol. 2010;21 Suppl 5:v103-15.

2. Vansteenkiste J, Crinò L, Dooms C, Douillard JY, Faivre-Finn C, Lim E, Rocco G, Senan S, van Schil P, Veronesi G, Stahel R, Peters S, Felip E, Kerr K, Besse B, Eberhardt W, Edelman M, Mok T, O'Byrne K, Novello S, Bubendorf L, Marchetti A, Baas P, Reck M, Syrigos K, Paz-Ares L, Smit EF, Meldgaard P, Adjei A, Nicolson M, et al. 2nd ESMO consensus conference on lung cancer: Early-stage non-small-cell lung cancer consensus on diagnosis, treatment and follow-up. Ann Oncol. 2014;25:1462-74.

3. Paik S, Shak S, Tang G, Kim C, Baker J, Cronin M, Baehner FL, Walker MG, Watson D, Park T, Hiller W, Fisher ER, Wickerham DL, Bryant J, Wolmark N. A multigene assay to predict recurrence of tamoxifen-treated, node-negative breast cancer. N Engl J Med. 2004;351:2817-26.

4. Lee E-S, Son D-S, Kim S-H, Lee J, Jo J, Han J, Kim H, Lee HJ, Choi HY, Jung Y, Park M, Lim YS, Kim K, Shim Y, Kim BC, Lee K, Huh N, Ko C, Park K, Lee JW, Choi YS, Kim J. Prediction of recurrence-free survival in postoperative non-small cell lung cancer patients by using an integrated model of clinical information and gene expression. Clin Cancer Res. 2008;14:7397-404.

5. Zhu C-Q, Ding K, Strumpf D, Weir BA, Meyerson M, Pennell N, Thomas RK, Naoki K, Ladd-Acosta C, Liu N, Pintilie M, Der S, Seymour L, Jurisica I, Shepherd FA, Tsao M-S. Prognostic and predictive gene signature for adjuvant chemotherapy in resected non-small-cell lung cancer. J Clin Oncol. 2010:28:4417-24.

6. Shedden K, Taylor JMG, Enkemann SA, Tsao M-S, Yeatman TJ, Gerald WL, Eschrich S, Jurisica I, Giordano TJ, Misek DE, Chang AC, Zhu CQ, Strumpf D, Hanash S, Shepherd FA, Ding K, Seymour L, Naoki K, Pennell N, Weir B, Verhaak R, Ladd-Acosta C, Golub T, Gruidl M, Sharma A, Szoke J, Zakowski M, Rusch V, Kris M, Viale A, et al. Gene expression-based survival prediction in lung adenocarcinoma: a multi-site, blinded validation study. Nat Med. 2008; 14:822-7

7. Rousseaux S, Debernardi A, Jacquiau B, Vitte A-L, Vesin A, Nagy-Mignotte $H_{\text {, }}$ Moro-Sibilot D, Brichon P-Y, Lantuejoul S, Hainaut P, Laffaire J, de Reyniès A, Beer DG, Timsit J-F, Brambilla C, Brambilla E, Khochbin S. Ectopic activation of germline and placental genes identifies aggressive metastasis-prone lung cancers. Sci Transl Med. 2013;5:186ra66.

8. Okayama H, Kohno T, Ishii Y, Shimada Y, Shiraishi K, Iwakawa R, Furuta K, Tsuta K, Shibata T, Yamamoto S, Watanabe S, Sakamoto H, Kumamoto K, Takenoshita S, Gotoh N, Mizuno H, Sarai A, Kawano S, Yamaguchi R, Miyano S, Yokota J. Identification of genes upregulated in ALK-positive and EGFR/ KRAS/ALK-negative lung adenocarcinomas. Cancer Res. 2012;72:100-11.

9. Botling J, Edlund K, Lohr M, Hellwig B, Holmberg L, Lambe M, Berglund A, Ekman S, Bergqvist M, Pontén F, König A, Fernandes O, Karlsson M, Helenius G, Karlsson C, Rahnenführer J, Hengstler JG, Micke P. Biomarker discovery in non-small cell lung cancer: integrating gene expression profiling, metaanalysis, and tissue microarray validation. Clin Cancer Res. 2013;19:194-204.

10. Der SD, Sykes J, Pintilie M, Zhu C-Q, Strumpf D, Liu N, Jurisica I, Shepherd FA, Tsao M-S. Validation of a histology-independent prognostic gene signature for early-stage, non-small-cell lung cancer including stage IA patients. J Thorac Oncol. 2014;9:59-64.

11. Eklund AC, Szallasi Z. Correction of technical bias in clinical microarray data improves concordance with known biological information. Genome Biol. 2008;9:R26.

12. Krzystanek M, Szallasi Z, Eklund AC. Biasogram: visualization of confounding technical bias in gene expression data. PLoS One. 2013;8:e61872.

13. Wistuba II, Behrens C, Lombardi F, Wagner S, Fujimoto J, Raso MG, Spaggiari L, Galetta D, Riley R, Hughes E, Reid J, Sangale Z, Swisher SG, Kalhor N, Moran CA, Gutin A, Lanchbury JS, Barberis M, Kim ES. Validation of a Proliferation-Based Expression Signature as Prognostic Marker in Early Stage Lung Adenocarcinoma. Clin Cancer Res. 2013;19:6261-71.

14. Bueno R, Hughes E, Lanchbury JS, Gustafson C, Jones JT, Barberis M, Wistuba I, Wallace WA, Harrison DJ. Validation of a Molecular and Pathological Model for Five-Year Mortality Risk in Patients with Early Stage Lung Adenocarcinoma. J Thorac Oncol. 2015;10:67-73.

15. Li B, Dewey CN. RSEM: accurate transcript quantification from RNA-Seq data with or without a reference genome. BMC Bioinformatics. 2011;12:323. 
16. Network TCGAR. Comprehensive molecular profiling of lung adenocarcinoma. Nature. 2014;511:543-50.

17. Carter SL, Eklund AC, Kohane IS, Harris LN, Szallasi Z. A signature of chromosomal instability inferred from gene expression profiles predicts clinical outcome in multiple human cancers. Nat Genet. 2006;38:1043-8.

18. Paik S. Development and clinical utility of a 21-gene recurrence score prognostic assay in patients with early breast cancer treated with tamoxifen. Oncologist. 2007;12:631-5.

19. Gentles AJ, Newman AM, Liu CL, Bratman SV, Feng W, Kim D, Nair VS, Xu Y, Khuong A, Hoang CD, Diehn M, West RB, Plevritis SK, Alizadeh AA. The prognostic landscape of genes and infiltrating immune cells across human cancers. Nat Med. 2015;21:938-45.

20. Birkbak NJ, Eklund AC, Li Q, McClelland SE, Endesfelder D, Tan P, Tan IB, Richardson AL, Szallasi Z, Swanton C. Paradoxical relationship between chromosomal instability and survival outcome in cancer. Cancer Res. 2011;71:3447-52.

Submit your next manuscript to BioMed Central and we will help you at every step:

- We accept pre-submission inquiries

- Our selector tool helps you to find the most relevant journal

- We provide round the clock customer support

- Convenient online submission

- Thorough peer review

- Inclusion in PubMed and all major indexing services

- Maximum visibility for your research

Submit your manuscript at www.biomedcentral.com/submit
Biomed Central 